\title{
Pharmabiotic Manipulation of the Microbiota in Gastrointestinal Disorders: A Clinical Perspective
}

\author{
Fanny Giron and Eamonn M M Quigley* \\ Gastroenterology and Hepatology, Lynda K and David M Underwood Center for Digestive Disorders, Houston Methodist Hospital and Weill \\ Cornell Medical College, Houston, Texas, USA
}

The advent and widespread availability of high-throughput technology has revolutionized the assessment of the communities of microorganisms that inhabit the gastrointestinal tract--the gut microbiota. As our understanding of the role of the microbiota in health and human disease increases, so also do efforts to prevent and treat disease through the modulation of the microbiota. Several strategies are available to us and range from time honored approaches, such as antibiotics and probiotics, to changes in diet, the administration of prebiotics as food supplements, and fecal microbiota transplantation. Of these, diet is perhaps the most pervasive but often ignored modulator of the microbiota, and a failure to recognize its impact complicates the interpretation of many microbiota studies. The impacts of antibiotics on the microbiota are more complex than originally thought and, though antibiotics can be life-saving, their effects on commensal bacterial populations can be clinically significant. Though there have been many studies of, and even more claims made for, probiotics, the majority of available studies suffer from significant deficits in study design and execution and many claims remain to be substantiated. Though holding much promise, the study of prebiotics in human disease is still in its infancy. Possibilities other than the administration of live organisms have been identified through efforts to mine the microbiota for novel therapeutics and include: dead organisms, bacterial components, small molecules elaborated by bacteria, and even bacterial DNA. Accordingly, the term pharmabiotic has been introduced to encompass the full range of therapeutic possibilities that the microbiota offers.

(J Neurogastroenterol Motil 2018;24:355-366)

Key Words

Anti-bacterial agents; Gastrointestinal microbiome; Prebiotics; Probiotics; Synbiotics

\section{Introduction}

The gut microbiome and its implications for health and disease have evolved rapidly over the last two decades to become among the hottest areas of medical research. The advent, and widespread application, of high-throughput sequencing technology has facilitated the performance of many studies of the gut microbiota in health and disease, and led to the description of associations between microbial signatures and disease states. The advent of technologies such as metagenomics and metabolomics now permits a move from mere description of microbiota composition to the prediction of microbial activity and the definition of microbial products. This evolution in technology has unlocked the door to the development of therapeutic strategies based, not only on the modification of the microbiota or the modulation of it activities, but also on strategies that "mine" the microbiota for novel therapeutics. As clinicians, our primary goal is to translate this science into clinical practice by contributing to the

Received: January 8, 2018 Revised: March 4, 2018 Accepted: March 26, 2018

(c) This is an Open Access article distributed under the terms of the Creative Commons Attribution Non-Commercial License (http://creativecommons. org/licenses/by-nc/4.0) which permits unrestricted non-commercial use, distribution, and reproduction in any medium, provided the original work is properly cited.

*Correspondence: Eamonn M M Quigley, MD, FRCP, FACP, MACG, FRCPI

Division of Gastroenterology and Hepatology, Houston Methodist Hospital, 6550 Fannin St, SM1201, Houston, Texas 77030, USA Tel: +1-713-441-0853, Fax: +1-713-797-0622, E-mail: equigley@houstonmethodist.org 
diagnosis, prognosis and treatment of disease; in most instances, this remains premature. Thus, despite the rapid pace of progress, our understanding of the composition of the "normal" gut microbiome remains incomplete. Furthermore, despite truly groundbreaking developments in the laboratory, clinical trials of strategies to modulate the microbiota in man still lag behind and often suffer from limitations in study design. ${ }^{1}$ In this review we will assess the current status and clinical implications of those approaches that attempt to modulate the human gut microbiome. Before we evaluate these therapeutic strategies let us briefly take stock of what we know of the gut microbiome in health and disease.

\section{The Gut Microbiome in Health and Disease: What Do We Know?}

The nature and the importance of the complex interactions between the microbiome and its host are now well recognized and the contributions of this commensal relationship to the health of the host increasingly appreciated. Accordingly, one can begin to postulate how the disruption of this relationship can lead to pathological consequences for the host. ${ }^{2}$

One of the most striking and best-studied illustrations of the clinical consequences of the disruption of this homeostasis is Clostridium difficile infection (CDI)-C. difficile-associated disease (CDAD). We are in the midst of a veritable epidemic of CDAD, and the mechanism(s) by which a course of antibiotics render the host susceptible to CDI are beginning to be understood. It would appear that an intact native microbiota provides colonization resistance against CDI; this protection is lost as a result of the bystander effects of antibiotics on the commensal microbiota rendering the host vulnerable to the development of antibiotic associated diarrhea and, of course, CDAD. ${ }^{3}$ Several mechanisms whereby an intact gut microbiota provides colonization resistance against CDI have been identified and include:

- The deconjugation of bile acids, gut bacteria promote the production of secondary bile acids which are inhibitory to $C$. difficile $^{4}$

- Niche exclusion through competition for nutrients ${ }^{5,6}$

- Production of bacteriocins by the intestinal microbiota that are effective against $C$. difficile ${ }^{3,7}$

- Toll-like receptor signaling ${ }^{3,8}$

The relative importance of these proposed mechanisms in the prevention of CDI and CDAD continues to be defined and, ultimately, could lead to innovative new treatments for CDI. The science behind CDI and CDAD illustrates several fundamental aspects of gut microbial biology; each may well be relevant to microbe-host interactions in other disease states.

Changes in the luminal microbiome could also contribute to the pathogenesis of a very common disease worldwide-gastroesophageal reflux disease (GERD) and its most feared complication, esophageal adenocarcinoma, whose incidence has increased significantly in the United States in recent decades. ${ }^{9}$ Yet, the cause(s) of GERD and the pathway(s) whereby it progresses to cancer remain largely unknown; now comes the suggestion that the microbiome may be relevant. ${ }^{10}$ Samples for microbiota analysis were obtained from control subjects, as well as patients with GERD and Barrett's esophagus. Microbiological analyses, performed blind to the source of the samples, identified 2 distinct microbial signatures: type I, dominated by Gram positive aerobic organisms and thought to represent a direct extension of the normal oral microbiome into the esophagus via swallowed saliva, and type II, dominated by Gram negative anaerobes and hypothesized to represent the regurgitation of gastric and intestinal bacteria into the esophagus in refluxed gastric juice. Interestingly, the type I microbiome was associated with those who had a phenotypically normal esophagus whereas the type II microbiome was strongly associated with esophagitis and Barrett's esophagus. Others have also reported alterations in esophageal microbial populations in gastroesophageal reflux and Barrett's esophagus. ${ }^{11,12}$ These findings do not establish cause and effect; thus it could be concluded that acid suppressive therapy promoted these changes in the gastric microbiome through its alteration of the luminal milieu or that gastroesophageal reflux modifies the esophageal microbiome by selecting against acid sensitive bacteria in the esophagus. However, though proton pump inhibitors have been shown to alter the microbiome, ${ }^{13,14}$ others have described an altered gastric microbiome in GERD independent of PPI use. ${ }^{13}$ Alternately, a more direct effect of Gram negative organisms could be invoked - inflammation and alterations in esophageal physiology induced by lipopolysaccharides, for example, could promote inflammation and lay the groundwork for the development of Barrett's esophagus and its progression to adenocarcinoma (Fig. 1). ${ }^{15}$

Gut microbiota alterations have also been associated with metabolic conditions such as diabetes, disorders of considerable interest to the field of neurogastroenterology, given the prevalence of motility disorders such as gastroparesis among affected individuals. ${ }^{16}$ Based on the characterization of the fecal metagenome of women with normal glucose homeostasis, impaired glucose tolerance or overt diabetes, a mathematical model linking the metagenome to diabetes was developed. When applied to a separate exploratory population comprised of females with impaired glucose tolerance 
Type I Esophageal microbiome

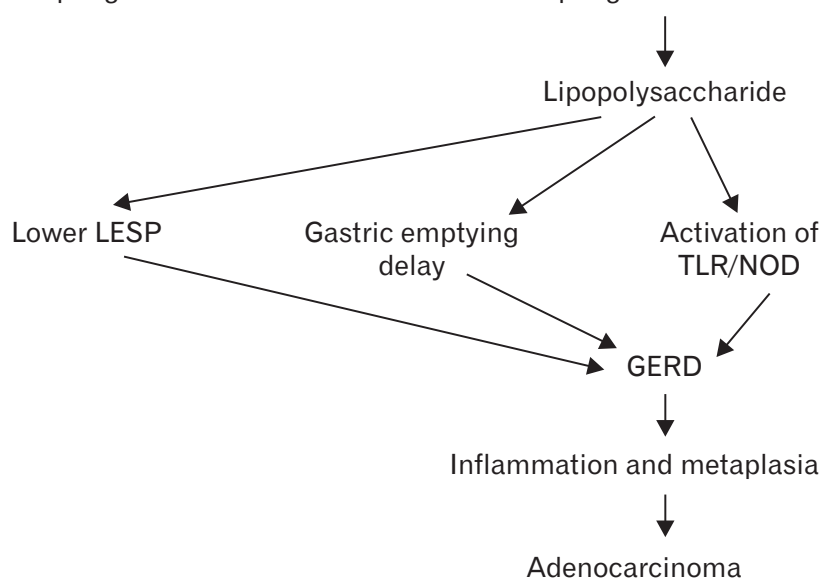

Figure 1. The esophageal microbiome, gastroesophageal reflux disease (GERD), Barrett's esophagus, and esophageal adenocarcinoma--an hypothesis. ${ }^{11}$ A shift towards a more Gram negative microbiome who produce more lipopolysaccharide (LPS) negatively impacts on gastro-esophageal function and promotes inflammation, a precursor of metaplasia, dysplasia, and cancer. LESP, lower esophageal sphincter pressure; TLR, Toll-like receptor; NOD, nucleosidebinding oligomerization domain-like.

the model proved to be highly predictive of progression to type 2 diabetes. ${ }^{17}$ Indeed, in health, certain bacterial taxa contribute to the post-prandial blood sugar response to everyday meals, ${ }^{18} \mathrm{a}$ finding of considerable interest to the gastroenterologist given the known interactions between glycemia, gastric emptying rate, and gastric neurophysiology. ${ }^{19}$ Furthermore, the efficacy of metformin in diabetes and pre-diabetes may be related in part to its effects on the microbiota ${ }^{20,21}$; this concept of a role for the microbiota in the clinical response to pharmacological agents may well extend to other therapeutic classes.

These findings in diabetes, facilitated by the availability of molecular methods paired with advances in informatics, provide an example of how microbial signatures may lead to the identification of disease predictors and therapeutic responses and, thereby, contribute to the early detection, prevention and optimal therapy of certain diseases. ${ }^{22}$ Non-alcoholic fatty liver disease (NAFLD), a relatively new disorder that has rapidly evolved to become a true epidemic in many countries around the world and can progress, in some, to complications such as cirrhosis and hepatocellular carcinoma, provides another example. Hence, the quest for a noninvasive method for the early detection of fibrosis; the harbinger of progressive disease in individuals with NAFLD. ${ }^{23}$ It appears that certain bacterial species are associated with advanced fibrosis and, thus, predictive of progressive disease. While the gut microbiome in NAFLD without advanced features was predominated by Gram positive bacteria (predominantly Firmicutes), progression to advanced fibrosis was linked to a shift to Gram negative bacteria (predominantly Proteobacteria, including Escherichia coli). A model that included these findings demonstrated highly robust diagnostic accuracy for detecting advanced fibrosis ${ }^{24}$; suggesting that this microbial signature may serve as a biomarker to predict disease progression in NAFLD. Here again, there is relevance to neurogastroenterology. Small intestinal bacterial overgrowth (SIBO) has been recognized in liver disease, in general, for decades and implicated in the development of various complications of cirrhosis through the occurrence of bacterial translocation promoted through an impaired gut barrier. ${ }^{25,26}$ Impaired gut motility has been shown to play an important role in SIBO pathogenesis in liver disease. ${ }^{27}$ In experimental models of NAFLD, SIBO has been shown to promote both steatosis and inflammation $^{28}$ and, in clinical studies, SIBO has been linked to nonalcoholic steatohepatitis. ${ }^{29-31}$

Functional gastrointestinal disorders (FGIDs) and irritable bowel syndrome (IBS), in particular, may also have a microbiological basis. ${ }^{32,33}$ This is not a new idea and dates back decades to the observation that IBS could develop for the first time after a bacterial gastroenteritis. ${ }^{34}$ Though several studies have examined the status of the small intestinal, fecal and colonic microbiota in IBS, and while the results of these studies are far from consistent, it is evident that some IBS patients, at least, do harbor an altered microbiota. ${ }^{35}$ Even more fascinating is the observation that the fecal microbial signal may predict severity in IBS. ${ }^{36}$ In the very near future, further studies of large populations of IBS and FGIDs, in general, which take account of the heterogeneity of these disorders and the presence of a variety of confounding factors, should reveal the true status of the microbiome in pathogenesis.

\section{Problems With Clinical Studies of the Microbiome in Disease}

In a highly selective sampling of the recent literature we have illustrated how a disrupted microbiome may result in intestinal disease and provided some examples of how microbiome signatures may define prognosis and predict response to therapy. Many, many other studies have described associations between an altered microbiome and various gastrointestinal and systemic diseases and disorders. Most of these studies, regrettably, share one or more of the following limitations ${ }^{1}$ :

1. Such is the heterogeneity in bacterial populations between 
and within patient populations that it is still not possible to state with certainty what is normal in any given population.

2. Most studies are single point in time, rather than longitudinal, rendering it impossible to account for fluctuations in disease activity, as well as the confounding impact of therapy. In other words, it can be nigh impossible to decide whether a given microbial signature represents state or trait in relation to a given disease. Only longitudinal studies with sampling at multiple time points can aid in making this distinction.

3. Diet, likely to be altered in many disease states, can significantly modify the microbiome (in both the long- and shortterm) and has not been accounted for in many of the studies.

4. For obvious reasons of convenience, most human studies have been based on the analysis of fecal samples, an approach that disregards variations in bacterial populations along the length of the gastrointestinal tract and may fail to represent those bacterial populations that reside close to, or adherent to, the mucosa.

Several studies have amply illustrated the impact of diet on the microbiome. In a study of elderly individuals in various residential settings, Claesson and colleagues ${ }^{37}$ demonstrated, not only a clear correlation between poor diet (ie, high fat/low fiber) and decreased microbial diversity, but were also able to link these changes to inflammation and poor health outcomes.

The same investigators looked at the impact of diet in a very different population, elite athletes. Though body mass index (BMI) was high in these athletes, this was attributable to muscle bulk and their body fat was very low. In comparison to both lean individuals and a group with who had similar BMIs to the athletes but possessed high body fat content, bacterial diversity was significantly higher in the athletes and, on principal coordinate analysis, the athletes' microbiota separated from both the low and high BMI control groups. This difference was mainly driven by protein intake, which accounted for considerably more of total energy intake in athletes, than in either control groups. ${ }^{38}$

\section{Microbiota Modulation: Clinical Impacts}

Several approaches may be taken to modulate the gut microbiome ranging from diet, through prebiotics and probiotics, antibiotics and on to fecal microbiota transplantation. We will briefly describe the role of, and recent innovations in, some of these areas.

\section{Diets and Food Supplements}

We have seen how changes in dietary habits modify the gut's bacterial diversity and function. Given the role of food in the precipitation of symptoms in FGIDs and the popularity of dietary modifications in their therapy, the impact of diet on the microbiota is of increasing interest to the neuro-gastroenterologist. Diets commonly recommended to FGID sufferers whether excluding a single dietary component (eg, lactose-, fructose-, and sorbitolfree diets), or involving more extensive modifications (eg, glutenfree diet, a low fermentable oligo-, di-, or mono-saccharides, and polyol [FODMAP's] diet, the Mediterranean or paleo diet), are likely to alter the composition of microbiota. For example, healthy individuals who consumed a gluten-free diet for 1 month developed changes in microbiome diversity and immunologic function: important commensals (Bifidobacterium and Lactobacillus) decreased and potential pathogens (Enterobacteriaceae) increased on the gluten-free diet. ${ }^{39}$ These changes correlated with a decreased intake of polysaccharides; dietary components which represent a major source of energy for commensal bacteria. ${ }^{39}$

A diet low in FODMAPs has been steadily gaining in popularity among patients with IBS and its utility is supported by positive results from clinical trials. ${ }^{40,41}$ Its long-term implementation has raised concerns about the impact of the exclusion of FODMAPs on important commensal bacterial populations. For example, oligosaccharides exert prebiotic effects ${ }^{42,43}$; consequently the growth of "good" bacteria may be compromised by the decreased intake of these dietary components. FODMAPs also serve as substrates for bacterial fermentation, yielding short chain fatty acids (SCFAs) which are an energy source for colonic epithelial cells. ${ }^{44}$ Shortterm results are reasonably reassuring. ${ }^{45}$ In comparison to a typical Australian diet, a low FODMAP diet was associated with a higher fecal $\mathrm{pH}$ but the concentration of SCFAs was similar between the 2 diets. On the low FODMAP diet total bacterial abundance was reduced while an increase in the relative abundance of butyrate-producing Clostridium cluster XIVa and mucus-associated Akkermansia muciniphila was observed among those on the typical Australian diet. These authors concluded that, while a low FODMAP diet reduced total bacterial abundance, there was no effect on the relative abundance of bacteria associated with colonic health. ${ }^{45}$ In another study that again confirmed the efficacy of the low FODMAP diet, ${ }^{46}$ changes in bacterial populations (a significant reduction in overall species richness with an increase in Actinobacteria) and metabolic activity raised concerns that this diet could expose a niche which could be occupied by potentially pathogenic bacteria. While the microbial repercussions of using a low FODMAP diet in the long-term are unknown, strategies are being explored that might mitigate deleterious effects on the microbiota. 
Could a probiotic prevent or reverse the effects of low FODMAP diet on the microbiome? In a placebo controlled study of the effects of a low FODMAP diet on IBS symptoms and the fecal microbiota, the abundance of Bifidobacterium species was decreased on the low FODMAP diet alone in comparison to subjects given the sham diet alone, but was restored to normal levels in subjects supplemented with a probiotic product. ${ }^{47}$

There is also evidence to suggest that the composition and metabolic activity of the microbiome may predict responses to the low FODMAP diet. In one study, children who responded to the low FODMAP diet featured a baseline microbiota rich in operational taxonomic units with greater saccharolytic activity (Bacteroides, $R u$ minococcus, and Dorea) compared to non-responders, who at baseline where enriched with members of the genus Turicibacter, which have lesser saccharolytic capacity and, therefore, are not well adapted to ferment the carbohydrates present in the low FODMAP diet. ${ }^{48}$ Bennet and colleagues ${ }^{49}$ examined fecal microbiota profiles before and after a low FODMAP and a traditional IBS diet. Pretreatment profile was predictive of response to a low FODMAP but not the traditional diet. In terms of treatment effects, the low FODMAP diet increased the Dysbiosis Index in some patients and reduced the populations of Bifidobacteria and Actinobacteria; these potentially negative effects were not seen with the traditional IBS diet. $^{49}$

Other dietary approaches that modulate the microbiota include the administration of dietary fiber or supplements with prebiotics: ie, foods that stimulate the growth of bacteria that are considered beneficial to the host (such as Lactobacilli and Bifidobacteria).

The term dietary fiber refers to a heterogeneous group of carbohydrate polymers of varying chemical composition and resultant solubility, fermentability and viscosity that, by definition, are neither digested nor absorbed in the human small intestine. ${ }^{50}$ The physicochemical properties of fiber are highly relevant to their biological and clinical impact; for example, soluble but not insoluble fibers have shown efficacy in IBS. ${ }^{51}$ While we cannot digest fibers our microbiome certainly can and, in so doing, produces SCFAs and gases; metabolic products that may contribute to their clinical effects and side effects. Fibers are an important source of nutrition for the colonic microbiome and a diet containing a range of fiber types has been shown to promote bacterial diversity. ${ }^{52}$

Among the various prebiotics that have been studied for effects in man are inulin, galactooligosaccharides, fructooligosaccharides, and xylooligosaccharides. ${ }^{53}$ These produce variable effects on the gut microbiota, both in terms of bacterial diversity and metabolism. ${ }^{54}$ Some prebiotics have been designed to be quite selective in terms of the bacterial species whose growth they stimulate- others have more general effects. ${ }^{55}$ While there is a lot of interest in the biological and clinical effects of prebiotics in man, there have been few studies of prebiotics administered either alone or in combination with a probiotic (referred to as a synbiotic) in gastrointestinal diseases. Prebiotics offer considerable promise and are safe; expect much more clinical research in this area in the coming years.

\section{Antibiotics}

Antibiotics targeted at a pathogen will inevitably and, to a greater or lesser extent, modify the commensal bacterial populations of the gastrointestinal tract. Thus, antibiotic therapy can reduce overall bacterial diversity by approximately a third, ${ }^{56}$ an effect that varies between different antibiotics. For example, in a cross-sectional study of elderly subjects, it was found that the levels of Bifidobacterium spp. were reduced 7-fold among those who had received an antibiotic in the prior month. There was a lesser impact in the relative abundance of nine genera including Lactobacillus spp. ${ }^{57}$

What is now evident is that even short-term courses of antibiotics can inflict long term sequelae on the gut microbiota. In an analysis of fecal samples from individuals prescribed clindamycin for 7 days, the collective number of Bacteroides clone types was reduced in all individuals exposed to the antibiotic. This reduction in diversity persisted for up to 2 years following antibiotic administration; in contrast to the control group where only minor variations in the number of clone types were seen. ${ }^{58}$

It is well known that low dose antibiotics function as growth promoters in animal husbandry. ${ }^{59}$ It is likely that those who eat meat from animals reared in this fashion will be exposed to very low doses of antibiotics; concerns have been raised on implications for human health. To assess how these sub-therapeutic doses of antibiotics comparable to those that we are likely to access through the food chain affect gut microbiome composition and metabolism mice were exposed at weaning to low doses of various antibiotics or a control substance through their drinking water. Even at these very low doses significant shifts in the taxonomic composition of the microbiome were observed and were associated with an increase in total fat mass. This was attributed to an increase in luminal and portal vein SCFA levels resulting in the upregulation of pathways involved in lipogenesis and triglyceride synthesis in the liver. ${ }^{60}$

\section{The Interesting History of Rifaximin in Irritable Bowel Syndrome}

Rifaximin, a poorly absorbed antibiotic, originally developed for the treatment of enteric infections, has been approved for the 
management of non-constipated IBS in the United States and elsewhere. Following encouraging results in phase II studies, 2 doubleblind, placebo-controlled pivotal phase III trials were conducted in 1260 patients with non-constipated IBS. ${ }^{61}$ Those on rifaximin were more likely to experience adequate relief of global IBS symptoms (40.7\% vs $31.7 \%$ for placebo) and of bloating (40.2\% vs $30.3 \%$ ). What is interesting in this and prior studies is that rifaximin given, albeit in a high dose (550 mg 3 times a day), for just 2 weeks can provide symptom relief for up to 10 weeks. ${ }^{61,62}$ This effect was reproducible on re-treatment. ${ }^{63}$ In all of these studies the safety profile of rifaximin was favorable and instances of $C$. difficile infection very rare $^{61-63}$ and, so far, the induction of antibiotic resistance has not been an issue. ${ }^{64}$

While one could argue about the clinical relevance of a therapeutic gain of around $10 \%$ for an expensive antibiotic in a select IBS population, the most compelling question is: why does it work? ? $^{64}$

The original hypothesis, namely the elimination of SIBO, is unlikely to explain most of the effect as the true prevalence of SIBO in IBS is probably low, ${ }^{65,66}$ none of the pivotal studies reported on its presence or contribution to the benefits of rifaximin ${ }^{61-63}$ and other studies have indicated that this antibiotic may reduce symptoms in IBS in the absence of a positive breath test at baseline. ${ }^{67}$ It must be conceded that other studies have shown that the presence of SIBO was a predictor of response to rifaximin ${ }^{68}$ and norfloxacin. ${ }^{69}$ While the status of SIBO in IBS remains to be conclusively defined, it seems reasonable to ask whether rifaximin could have other effects on the small intestinal or colonic microbiota relevant to its efficacy in IBS. Regrettably, there have been limited studies on the impact of rifaximin on the microbiome. Those that have been performed have failed to identify significant changes in diversity or operational taxonomic units abundance. ${ }^{70}$ Studies in models of inflammatory bowel disease suggest that rifaximin exerts an anti-inflammatory effect through its role as a human pregnane $\mathrm{X}$ activator. ${ }^{71}$

While much more detailed studies of the gut microbiome and its metabolic functions are warranted in IBS, some clues to its effects may be gleaned from studies in another condition for which rifaximin is approved and other antibiotics have been used for over half a century-hepatic encephalopathy. ${ }^{72}$ Experiments involving the "humanization" of germ free mice with stool from patients with mild hepatic encephalopathy revealed a number of effects of rifaximin that could contribute to benefits in encephalopathy. ${ }^{73}$ First, rifaximin reduced intestinal ammonia generation in the absence of the gut microbiota through a suppression of small intestinal glutaminase. Second, it improved gut barrier function and reduced the systemic inflammatory response to the cirrhotic microbiota by alter- ing microbiota function (decreased endotoxin formation and bile acid deconjugation) in the absence of any significant changes in its composition. $^{73}$

\section{Probiotics: The Emerging Science}

While the shelves of our pharmacies and supermarkets groan under the weight of products that claim to possess probiotic properties-which, by definition, implies that they confer a health benefit, ${ }^{74}$ very few can support such claims with high quality clinical evidence. Many do not even meet basic standards of quality control. Thanks to progress in technology it is now possible to characterize a putative probiotic at the most basic level — its genome and to, thereby, begin to predict its biology. The power of this approach is exemplified by recent work on Bifidobacterium longum subsp. longum $356244^{75}$ a probiotic widely used in the management of IBS-type symptoms. In describing the complete genome of this bacterium, investigators identified a specific gene cluster which encoded the machinery necessary for the synthesis of the lush exopolysaccharide (EPS) coat that envelops the organism ${ }^{75}$ and that appears to be essential to its anti-inflammatory activity. ${ }^{76}$

Another fundamental component of the current definition of a probiotic is that it is a live organism; ${ }^{77}$ in other words, it should survive transit through the gastrointestinal tract. ${ }^{78}$ Once probiotic administration ceases, it will, typically, no longer be recoverable in feces after a few weeks; thus for continued effects, long-term intake is required. ${ }^{79}$

In characterizing a probiotic and before embarking on human studies, it is essential to define its biological effects, be they, antibacterial, anti-inflammatory, immune modulating, or metabolic, to mention but a few. ${ }^{77,79-81}$ Appropriate models are available for such studies; blind guesses and empirical trials of randomly selected strains are no longer appropriate.

The impact of probiotics on the immune system has been of particular interest and probiotics have been shown to possess a variety of immunomodulatory properties such as enhancing immunoglobulin A production, down-regulating proinflammatory cytokines and, through engagement with dendritic cells (DCs), inducing regulatory $\mathrm{T}$ cells. It is now evident that the immune system can differentiate between a commensal (and, pari passu, a probiotic) and a pathogen. Instead of activating the nuclear factor-kappa $B$ $(\mathrm{NF}-\kappa \mathrm{B})$ inflammatory cascade which typifies the response to a pathogen, a commensal organism engages with DCs to activate a regulatory $\mathrm{T}$-cell pathway which is anti-inflammatory and leads to tolerance to the organism (Fig. 2). ${ }^{81-87}$

These effects have been seen in healthy human volunteers. ${ }^{83}$ 
Not only did the administration of a probiotic result in enhanced systemic secretion of the anti-inflammatory cytokine IL-10, but the same probiotic (a B. longum) was shown to stimulate IL-10 production by human DCs. ${ }^{83}$ That such effects might be clinically relevant was subsequently demonstrated by the ability of the same probiotic strain to reduce circulating levels of C-reactive protein among patients with a number of common inflammatory disorders. ${ }^{88}$ The clinical impact of probiotics on gastrointestinal disorders is beyond the scope of this review. Somewhat surprisingly, given the aforementioned description of anti-inflammatory and immune modulating properties, the impact of probiotics in inflammatory bowel disease has been, with the exception of pouchitis, modest. ${ }^{89-91}$ In contrast, certain probiotics have shown efficacy in IBS and in component symptoms of IBS, such as bloating and abdominal pain. ${ }^{89,92,93}$ Ultimately, recommendations for the use of probiotics in any gastrointestinal disorder must be based on evidence of the highest quality - large, appropriately powered randomized placebo controlled clinical trials. Regrettably, these remain few in number in this arena.

\section{The Pharmabiotic Concept}

This term has been introduced to encompass any biological entity mined from the microbiota that has the capacity to influence the microbiota in a manner that could be of therapeutic benefit. ${ }^{94}$ Thus this concept goes beyond live organisms, to dead organisms, bacterial components and bacterial products. EPS produced by $B$. longum subsp. longum, described above, provides one example of a potential pharmabiotic. In subsequent studies, investigators went on to develop a mutant strain lacking the EPS gene cluster which resulted, not only in the loss of the EPS coat, but also transformed a formerly anti-inflammatory bacterium into a pro-inflammatory one. ${ }^{76}$ This work, not only points the way towards the future of probiotic work, but also illustrates the uniqueness of every single probiotic strain - in this instance, the loss of just a single gene cluster completely transformed the function of the Bifidobacterium. A clear warning of the dangers of extrapolating from observations in

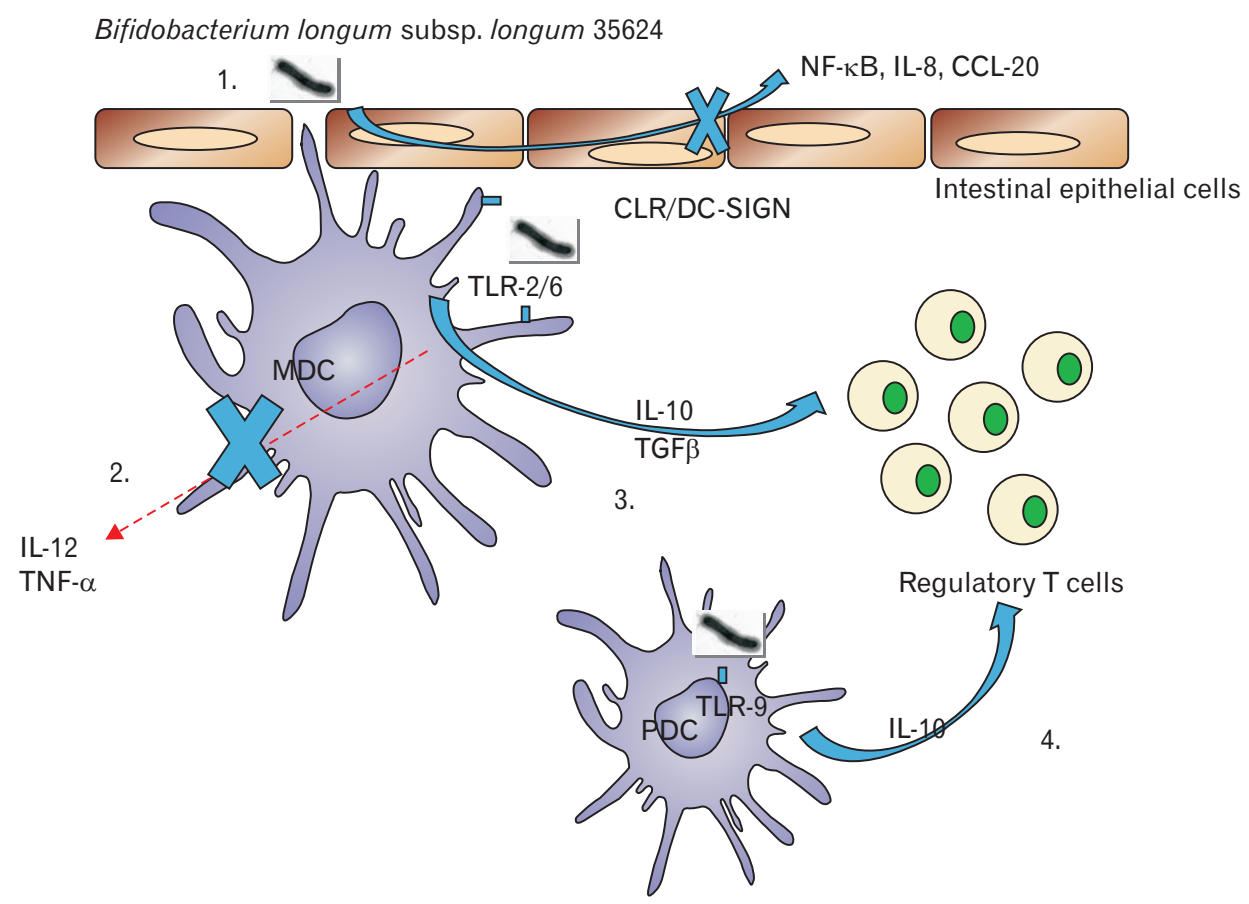

Figure 2. Engagement between the gut immune system and a probiotic (exemplified by Bifidobacterium longum subsp. longum 35624) leads to a regulatory T-cell response. ${ }^{57} 1$. Rather than initiating an inflammatory response via the nuclear factor-kappa B (NF- $\kappa$ B) pathway, B. longum subsp. longum 35624 engages with dendritic cells (DCs) through toll-like receptors (TLR) 2 and 6. 2. This does not lead to the release of inflammatory cytokines such as TNF- $\alpha$ and IL-12 which would typify engagement with a pathogen. 3. Instead, engagement with $B$. longum subsp. infantis 35624 leads to the secretion of the anti-inflammatory cytokines IL-10 and TGF- $\beta$ and the stimulation of regulatory T-cells. 4. Engagement of B. longum subsp. infantis 35624 via TLR-9 also stimulates IL-10 secretion and regulatory T-cell proliferation. CCL-20, chemokine (C-C motif) ligand 20; CLR, C-type lectin receptors; DC-SIGN, dendritic cell-specific intercellular adhesion molecule-3-grabbing non-integrin; MDC, myeloid-derived dendritic cells; PDC, plasmacytoid dendritic cells. Modified from Konieczna et a ${ }^{84}$ with permission. 
one strain or species to another!

Bacteriocins, antibacterial molecules produced by bacteria provide a well-established illustration of a pharmabiotic. Lactobacillus salivarius UCC118, for example, produced a bacteriocin, Abp118, which protected mice against infection with Listeria monocytogenes, an effect that was not achieved by a mutant $L$. salivarius strain devoid of the bacteriocin. ${ }^{95}$ Thuricin, a bacteriocin derived from Bacillus thuringienesis DPC 6431 is highly effective against $C$. difficile, yet has little effects on the commensal microbiome. ${ }^{7}$ Lately, the therapeutic potential of bacteriophages has been recognized ${ }^{96}$ and studies are under way to test the efficacy of a bacteriophage directed against adherent invasive $E$. coli strains in Crohn's disease. ${ }^{97}$ The gut microbiota may yet yield a bountiful harvest well beyond what is currently included within the definition of a probioticdead/attenuated organisms, ${ }^{98}$ genetically manipulated/modified organisms, ${ }^{99}$ other therapeutically active small molecules produced by bacteria, ${ }^{100,101}$ and biologically active bacterial components. ${ }^{102-104}$ For example, bacteria secrete neuroactive compounds ${ }^{101}$ and, in an animal model, even bacterial DNA was shown to exert potentially valuable biological effects. ${ }^{105}$ The more we learn about the most fundamental aspects of bacterial genomics and metabolism, ${ }^{106}$ as well as how these organisms interact with each other, their immediate environment and their living hosts, the sooner the full potential of pharmabiotics will be realized.

\section{Limitations to Human Studies of Microbiota Modulation in Man}

To date, clinical studies of most interventions that seek to modulate the microbiota leave a lot to be desired rendering their interpretation difficult and conclusions unsupported. Comparisons between probiotic studies, for example, are rendered nigh impossible by variations in strain selection, dosage, and delivery methods. Study design is equally problematic with many studies being underpowered and employing variable and often non-validated end points. Many potential confounders and, most notably diet, ${ }^{37-39,45-47,107}$ are ignored.

Good studies can be done but they are logistically challenging and expensive. The recent study on a synbiotic in early childhood sepsis shows the way-it is possible to complete an appropriately powered study and demonstrate a positive outcome. ${ }^{108}$

\section{The Future}

While many of the details remain to be filled in, it is evident that the gut microbiota plays a central role in homeostasis and may contribute to several diseases and disorders. Work in animal models has revealed the extent, as well as the complexity, of interactions between the commensal microbiota and its host. Progress in human studies continues, albeit at slower pace and limited by methodological shortcomings. The microbiota offers a veritable cornucopia of therapeutic possibilities yet our efforts to date have been largely limited to generally low quality studies of probiotics and a few studies of dietary changes, prebiotic supplements and synbiotics. The benefits of the full range of pharmabiotic possibilities has scarcely been tested despite the potential of genetically modified organisms, dead bacteria or bacterial products. This may well be the next frontier in microbiota therapeutics with modified bacteria delivering directly to their target therapeutic agents, vaccines and other molecules. Much can also be learned from the success of fecal microbiota transplantation - the identification of the bacterial strains or products essential for efficacy in a given disease state could pave the way to novel pharmabiotics.

\section{Financial support: None.}

Conflicts of interest: Consultant to Alimentary Health, Allergan, Biocodex, Ironwood, Menarini, Pharmasierra, Salix, Shire, Synergy, and Vibrant. Holds patents with and shares in Alimentary Health.

\section{References}

1. Quigley EMM. Gut microbiome as a clinical tool in gastrointestinal disease management: are we there yet? Nat Rev Gastroenterol Hepatol 2017;14:315-320.

2. Quigley EM. Gut bacteria in health and disease. Gastroenterol Hepatol (NY) 2013;9:560-569.

3. Britton RA, Young VB. Interaction between the intestinal microbiota and host in Clostridium difficile colonization resistance. Trends Microbiol 2012;20:313-319.

4. Ridlon JM, Kang DJ, Hylemon PB. Bile salt biotransformations by human intestinal bacteria. J Lipid Res 2006;47:241-259.

5. Merrigan MM, Sambol SP, Johnson S, Gerding DN. Prevention of fatal Clostridium difficile-associated disease during continuous administration of clindamycin in hamsters. J Infect Dis 2003;188:1922-1927.

6. Sambol SP, Merrigan MM, Tang JK, Johnson S, Gerding DN. Colonization for the prevention of Clostridium difficile disease in hamsters. J Infect Dis 2002;186:1781-1789.

7. Rea MC, Sit CS, Clayton E, et al. Thuricin CD, a posttranslationally modified bacteriocin with a narrow spectrum of activity against Clostridium difficile. Proc Natl Acad Sci USA 2010;107:9352-9357.

8. Lawley TD, Clare S, Walker AW, et al. Antibiotic treatment of Clostrid- 
ium difficile carrier mice triggers a supershedder state, spore-mediated transmission, and severe disease in immunocompromised hosts. Infect Immun 2009;77:3661-3669.

9. El-Serag HB. Time trends of gastroesophageal reflux disease: a systematic review. Clin Gastroenterol Hepatol 2007;5:17-26.

10. Yang L, Lu X, Nossa CW, Francois F, Peek RM, Pei Z. Inflammation and intestinal metaplasia of the distal esophagus are associated with alterations in the microbiome. Gastroenterology 2009;137:588-597.

11. Blackett KL, Siddhi SS, Cleary S, et al. Oesophageal bacterial biofilm changes in gastro-oesophageal reflux disease, Barrett's and oesophageal carcinoma: association or causality? Aliment Pharmacol Ther 2013;37:1084-1092.

12. Harris JK, Fang R, Wagner BD, et al. Esophageal microbiome in eosinophilic esophagitis. PLoS One 2015;10:e0128346.

13. Amir I, Konikoff FM, Oppenheim M, Gophna U, Half EE. Gastric microbiota is altered in oesophagitis and Barrett's oesophagus and further modified by proton pump inhibitors. Environ Microbiol 2014;16:2905-2914.

14. Freedberg DE, Toussaint NC, Chen SP, et al. Proton pump inhibitors alter specific taxa in the human gastrointestinal microbiome: a crossover trial. Gastroenterology 2015;149:883-885, e9.

15. Yang L, Francois F, Pei Z. Molecular pathways: pathogenesis and clinical implications of microbiome alteration in esophagitis and Barrett esophagus. Clin Cancer Res 2012;18:2138-2144.

16. Qin J, Li Y, Cai Z, et al. A metagenome-wide association study of gut microbiota in type 2 diabetes. Nature 2012;490:55-60.

17. Karlsson FH, Tremaroli V, Nookaew I, et al. Gut metagenome in European women with normal, impaired and diabetic glucose control. Nature 2013;498:99-103.

18. Zeevi D, Korem T, Zmora N, et al. Personalized nutrition by prediction of glycemic responses. Cell 2015;163:1079-1094.

19. Hayashi Y, Toyomasu Y, Saravanaperumal SA, et al. Hyperglycemia increases interstitial cells of Cajal via MAPK1 and MAPK3 signaling to ETV1 and KIT, leading to rapid gastric emptying. Gastroenterology 2017;153:521-535, e20.

20. Wu H, Esteve E, Tremaroli V, et al. Metformin alters the gut microbiome of individuals with treatment-naive type 2 diabetes, contributing to the therapeutic effects of the drug. Nat Med 2017;23:850-858.

21. Bauer PV, Duca FA, Waise TMZ, et al. Metformin Alters Upper Small Intestinal Microbiota that Impact a Glucose-SGLT1-Sensing Glucoregulatory Pathway. Cell Metab 2018;27:101-117, e5.

22. Aziz Q, Doré J, Emmanuel A, Guarner F, Quigley EM. Gut microbiota and gastrointestinal health: current concepts and future directions. Neurogastroenterol Motil 2013;25:4-15.

23. Angulo P, Kleiner DE, Dam-Larsen S, et al. Liver fibrosis, but no other histologic features, is associated with long-term outcomes of patients with nonalcoholic fatty liver disease. Gastroenterology 2015;149:389-397, e10.

24. Loomba R, Seguritan V, Li W, et al. Gut microbiome-based metagenomic signature for non-invasive detection of advanced fibrosis in human nonalcoholic fatty liver disease. Cell Metab 2017;25:1054-1062, e10.
25. Quigley EM. Gastrointestinal dysfunction in liver disease and portal hypertension. G-liver interactions revisited. Dig Dis Sci 1996;41:557-561.

26. Fukui H. Gut-liver axis in liver cirrhosis: How to manage leaky gut and endotoxemia. World J Hepatol 2015;7:425-442.

27. Theocharidou E, Dhar A, Patch D. Gastrointestinal motility disorders and their clinical implications in cirrhosis. Gastroenterol Res Pract 2017;2017:8270310.

28. Quigley EM, Monsour HP. The gut microbiota and nonalcoholic fatty liver disease. Semin Liver Dis 2015;35:262-269.

29. Wigg AJ, Roberts-Thomson IC, Dymock RB, McCarthy PJ, Grose RH, Cumming AG. The role of small intestinal bacterial overgrowth, intestinal permeability, endotoxemia, and tumor necrosis factor alpha in the pathogenesis of nonalcoholic steatohepatitis. Gut 2001;48:206-211.

30. Abu Shanab A, Scully P, Crosbie O, et al. Small intestinal bacterial overgrowth in non-alcoholic steato-hepatitis; association with toll-like receptor 4 expression and plasma levels of interleukin 8. Dig Dis Sci 2011;56:1524-1534.

31. Ghoshal UC, Baba CS, Ghoshal U, et al. Low-grade small intestinal bacterial overgrowth is common in patients with non-alcoholic steatohepatitis on quantitative jejunal aspirate culture. Indian J Gastroenterol 2017;36:390-399.

32. Quigley EM. Bugs on the brain; brain in the gut--seeking explanations for common gastrointestinal symptoms. Ir J Med Sci 2013;182:1-6.

33. De Palma G, Collins SM, Bercik P, Verdu EF. The microbiota-gutbrain axis in gastrointestinal disorders: stressed bugs, stressed brain, or both? J Physiol 2014;592:2989-2997.

34. Klem F, Wadhwa A, Prokop LJ, et al. Prevalence, risk factors, and outcomes of irritable bowel syndrome after infectious enteritis: a systematic review and meta-analysis. Gastroenterology 2017;152:1042-1054.

35. Liu HN, Wu H, Chen YZ, Chen YJ, Shen XZ, Liu TT. Altered molecular signature of intestinal microbiota in irritable bowel syndrome patients compared with healthy controls: A systematic review and metaanalysis. Dig Liver Dis 2017;49:331-337.

36. Tap J, Derrien M, Törnblom H, et al. Identification of an intestinal microbiota signature associated with severity of irritable bowel syndrome. Gastroenterology 2017;152:111-123, e8.

37. Claesson MJ, Jeffery IB, Conde S, et al. Gut microbiota composition correlates with diet and health in the elderly. Nature 2012;488:178-184.

38. Clarke SF, Murphy EF, O'Sullivan O, et al. Exercise and associated dietary extremes impact on gut microbial diversity. Gut 2014;63:19131920.

39. De Palma G, Nadal I, Collado MC, Sanz Y. Effects of a gluten-free diet on gut microbiota and immune function in healthy adult human subjects. Br J Nutr 2009;102:1154-1160.

40. Staudacher HM, Whelan K, Irving PM, Lomer MC. Comparison of symptom response following advice for a diet low in fermentable carbohydrates (FODMAPs) versus standard dietary advice in patients with irritable bowel syndrome. J Hum Nutr Diet 2011;24:487-495.

41. Halmos EP, Power VA, Shepherd SJ, Gibson PR, Muir JG. A diet low in FODMAPs reduces symptoms of irritable bowel syndrome. Gastroenterology 2014;146:67-75, e5.

42. Bouhnik Y, Flourié B, Riottot M, et al. Effects of fructo-oligosaccha- 
rides ingestion on fecal bifidobacteria and selected metabolic indexes of colon carcinogenesis in healthy humans. Nutr Cancer 1996;26:21-29.

43. Davis LM, Martinez I, Walter J, Hutkins R. A dose dependent impact of prebiotic galactooligosaccharides on the intestinal microbiota of healthy adults. Int J Food Microbiol 2010;144:285-292.

44. Cook SI, Sellin JH. Review article: short chain fatty acids in health and disease. Aliment Pharmacol Ther 1998;12:499-507.

45. Halmos EP, Christophersen CT, Bird AR, Shepherd SJ, Gibson PR, Muir JG. Diets that differ in their FODMAP content alter the colonic luminal microenvironment. Gut 2015;64:93-100.

46. McIntosh K, Reed DE, Schneider T, et al. FODMAPs alter symptoms and the metabolome of patients with IBS: a randomised controlled trial. Gut 2017;66:1241-1251.

47. Staudacher HM, Lomer MCE, Farquharson FM, et al. A Diet low in FODMAPs reduces symptoms in patients with irritable bowel syndrome and a probiotic restores Bifidobacterium Species: a randomized controlled trial. Gastroenterology 2017;153: 936-947.

48. Chumpitazi BP, Cope JL, Hollister EB, et al. Randomised clinical trial: gut microbiome biomarkers are associated with clinical response to a low FODMAP diet in children with the irritable bowel syndrome. Aliment Pharmacol Ther 2015;42:418-427.

49. Bennet SMP, Böhn L, Störsrud S, et al. Multivariate modelling of faecal bacterial profiles of patients with IBS predicts responsiveness to a diet low in FODMAPs. Gut 2018;67:872-881.

50. Codex Alimentarius Committee Guidelines on nutrition labelling CAC/ GL 2-1985 as last amended 2010. Joint FAO/WHO Food Standards Programme, Secretariat of the Codex Alimentarius Commission. Rome, Italy: FAO. 2010.

51. Moayyedi P, Quigley EM, Lacy BE, et al. The effect of fiber supplementation on irritable bowel syndrome: a systematic review and metaanalysis. Am J Gastroenterol 2014;109:1367-1374.

52. Cummings JH, Macfarlane GT. The control and consequences of bacterial fermentation in the human colon. J Appl Bacteriol 1991;70:443459.

53. Gibson GR, Hutkins R, Sanders ME, et al. Expert consensus document: the International Scientific Association for Probiotics and Prebiotics (ISAPP) consensus statement on the definition and scope of prebiotics. Nat Rev Gastroenterol Hepatol 2017;14:491-502.

54. Holscher HD. Dietary fiber and prebiotics and the gastrointestinal microbiota. Gut Microbes 2017;8:172-184.

55. Rastall RA, Gibson GR. Recent developments in prebiotics to selectively impact beneficial microbes and promote intestinal health. Curr Opin Biotechnol 2015;32:42-46.

56. Dethlefsen L, Huse S, Sogin ML, Relman DA. The pervasive effects of an antibiotic on the human gut microbiota, as revealed by deep $16 \mathrm{~S}$ rRNA sequencing. PLoS Biol 2008;6:e280.

57. O'Sullivan O, Coakley M, Lakshminarayanan B, et al. Alterations in intestinal microbiota of elderly Irish subjects post-antibiotic therapy. J Antimicrob Chemother 2013;68:214-221.

58. Jernberg C, Löfmark S, Edlund C, Jansson JK. Long-term ecological impacts of antibiotic administration on the human intestinal microbiota. ISME J 2007;1:56-66.
59. Butaye P, Devriese LA, Haesebrouck F. Antimicrobial growth promoters used in animal feed: effects of less well known antibiotics on grampositive bacteria. Clin Microbiol Rev 2003;16:175-188.

60. Cho I, Yamanishi S, Cox L, et al. Antibiotics in early life alter the murine colonic microbiome and adiposity. Nature 2012;488:621-626.

61. Pimentel M, Lembo A, Chey WD, et al. Rifaximin therapy for patients with irritable bowel syndrome without constipation. N Engl J Med 2011;364:22-32.

62. Gatta L, Scarpignato C. Systematic review with meta-analysis: rifaximin is effective and safe for the treatment of small intestine overgrowth. Aliment Pharmacol Ther 2017;45:604-616.

63. Lembo A, Pimentel M, Rao SS, et al. Repeat treatment with rifaximin is safe and effective in patients with diarrhea-predominant irritable bowel syndrome. Gastroenterology 2016;151:1113-1121.

64. Pimentel M, Cash BD, Lembo A, Wolf RA, Israel RJ, Schoenfeld P. Repeat rifaximin for irritable bowel syndrome: no clinically significant changes in stool microbial antibiotic sensitivity. Dig Dis Sci 2017;62:2455-2463.

65. Quigley EM. Antibiotics for irritable bowel syndrome: hitting the target, but what is it? Gastroenterology 2011;141:391-393.

66. Ghoshal UC, Srivastava D. Irritable bowel syndrome and small intestinal bacterial overgrowth: meaningful association or unnecessary hype. World J Gastroenterol 2014;20:2482-2491.

67. Sharara AI, Aoun E, Abdul-Baki H, Mounzer R, Sidani S, Elhajj I. A randomized double-blind placebo-controlled trial of rifaximin in patients with abdominal bloating and flatulence. Am J Gastroenterol 2006;101:326-333.

68. Zhao J, Zheng X, Chu H, et al. A study of the methodological and clinical validity of the combined lactulose hydrogen breath test with scintigraphic oro-cecal transit test for diagnosing small intestinal bacterial overgrowth in IBS patients. Neurogastroenterol Motil 2014;26:794802.

69. Ghoshal UC, Srivastava D, Misra A, Ghoshal U. A proof-of-concept study showing antibiotics to be more effective in irritable bowel syndrome with than without small-intestinal bacterial overgrowth: a randomized, double-blind, placebo-controlled trial. Eur J Gastroenterol Hepatol 2016;28:281-289.

70. Zeber-Lubecka N, Kulecka M, Ambrozkiewicz F, et al. Limited prolonged effects of rifaximin treatment on irritable bowel syndrome-related differences in the fecal microbiome and metabolome. Gut Microbes 2016;7:397-413.

71. Ma X, Shah YM, Guo GL, et al. Rifaximin is a gut-specific human pregnane X receptor activator. J Pharmacol Exp Ther 2007;322:391398.

72. Kimer N, Krag A, Møller S, Bendtsen F, Gluud LL. Systematic review with meta-analysis: the effects of rifaximin in hepatic encephalopathy. Aliment Pharmacol Ther 2014;40:123-132.

73. Kang DJ, Kakiyama G, Betrapally NS, et al. Rifaximin exerts beneficial effects independent of its ability to alter microbiota composition. Clin Transl Gastroenterol 2016;7:e187.

74. Hill C, Guarner F, Reid G, et al. Expert consensus document. The International Scientific Association for Probiotics and Prebiotics consensus 
ststement on the scope and appropriate use of the term probiotic. Nat Rev Gastroenterol Hepatol 2014;11:506-514.

75. Altmann F, Kosma P, O'Callaghan A, et al. Genome analysis and characterisation of the exopolysaccharide produced by Bifidobacterium longum subsp. longum 35624. PLoS One 2016;11:e162983.

76. Schiavi E, Gleinser M, Molloy E, et al. The surface-associated exopolysaccharide of Bifidobacterium longum 35624 plays an essential role in dampening host proinflammatory responses and repressing local TH17 responses. Appl Environ Microbiol 2016;82:7185-7196.

77. Dunne C, O'Mahony L, Murphy L, et al. In vitro selection criteria for probiotic bacteria of human origin: correlation with in vivo findings. Am J Clin Nutr 2001;73(2 suppl):386s-392s.

78. Charbonneau D, Gibb RD, Quigley EM. Fecal excretion of Bifidobacterium infantis 35624 and changes in fecal microbiota after eight weeks of oral supplementation with encapsulated probiotic. Gut Microbes 2013;4:201-211.

79. Collins JK, Dunne C, Murphy L, et al. A Randomised controlled trial of a probiotic Lactobacillus strain in healthy adults: assessment of its delivery, transit and influence on microbial flora and enteric immunity. Microb Ecol Health Dis 2002;14:81-89.

80. O'Mahony L, Feeney M, O'Halloran S, et al. Probiotic impact on microbial flora, inflammation and tumour development in IL-10 knockout mice. Aliment Pharmacol Ther 2001;15:1219-1225.

81. Sibartie S, O'Hara AM, Ryan J, et al. Modulation of pathogen-induced CCL20 secretion from HT-29 human intestinal epithelial cells by commensal bacteria. BMC Immunology 2009;10:54 .

82. O'Mahony L, O'Callaghan L, McCarthy J, et al. Differential cytokine response from dendritic cells to commensal and pathogenic bacteria in different lymphoid compartments in humans. Am J Physiol Gastrointest Liver Physiol 2006;290:G839-G845.

83. Konieczna P, Groeger D, Ziegler M, et al. Bifidobacterium infantis 35624 administration induces Foxp3 T regulatory cells in human peripheral blood: potential role for myeloid and plasmacytoid dendritic cells. Gut 2012;61:354-366.

84. Konieczna P, Akdis CA, Quigley EM, Shanahan F, O'Mahony L. Portrait of an immunoregulatory bifidobacterium. Gut Microbes 2012;3:261-266.

85. Symonds EL, O'Mahony C, Lapthorne S, et al. Bifidobacterium infantis 35624 protects against salmonella-induced reductions in digestive enzyme activity in mice by attenuation of the host inflammatory response. Clin Transl Gastroenterol 2012;3:e15.

86. Scully P, Macsharry J, O'Mahony D, et al. Bifidobacterium infantis suppression of Peyer's patch MIP-1alpha and MIP-1beta secretion during Salmonella infection correlates with increased local CD4+CD25+ T cell numbers. Cell Immunol 2013;281:134-140.

87. Konieczna P, Ferstl R, Ziegler M, et al. Immunomodulation by Bifidobacterium infantis 35624 in the murine lamina propria requires retinoic acid-dependent and independent mechanisms. PLoS One 2013;8:e62617.

88. Groeger D, O'Mahony L, Murphy EF, et al. Bifidobacterium infantis 35624 modulates host inflammatory processes beyond the gut. Gut Microbes 2013;4:325-339.
89. Shanahan F, Quigley EM. Manipulation of the microbiota for treatment of IBS and IBD-challenges and controversies. Gastroenterology 2014;146:1554-1563.

90. Abraham BP and Quigley EMM. Probiotics in inflammatory bowel disease. Gastroenterol Clin N Am 2017;46:769-782.

91. Derwa Y, Gracie DJ, Hamlin PJ, Ford AC. Systematic review with meta-analysis: the efficacy of probiotics in inflammatory bowel disease. Aliment Pharmacol Ther 2017;46:389-400.

92. Ford AC, Quigley EM, Lacy BE, et al. Efficacy of prebiotics, probiotics, and synbiotics in irritable bowel syndrome and chronic idiopathic constipation: systematic review and meta-analysis. Am J Gastroenterol 2014;109:1547-1561.

93. Hungin APS, Mitchell CR, Whorwell P, et al. Systematic review: probiotics in the management of lower gastrointestinal symptoms - an updated evidence-based international consensus. Aliment Pharmacol Ther 2018;47:1054-1070.

94. Shanahan F, Collins SM. Pharmabiotic manipulation of the microbiota in gastrointestinal disorders, from rationale to reality. Gastroenterol Clin North Am 2010;39:721-726.

95. Corr SC, Li Y, Riedel CU, O’Toole PW, Hill C, Gahan CG. Bacteriocin production as a mechanism for the antiinfective activity of Lactobacillus salivarius UCC118. Proc Natl Acad Sci USA 2007;104:76177621.

96. Łusiak-Szelachowska M, Weber-Dąbrowska B, Jończyk-Matysiak E, Wojciechowska R, Górski A. Bacteriophages in the gastrointestinal tract and their implications. Gut Pathog 2017;9:44.

97. Galtier M, De Sordi L, Sivignon A, et al. Bacteriophages targeting adherent invasive Escherichia coli strains as a promising new treatment for Crohn's disease. J Crohns Colitis 2017;11:840-847.

98. Liévin-Le Moal V. A gastrointestinal anti-infectious biotherapeutic agent: the heat-treated Lactobacillus LB. Therap Adv Gastroenterol 2016;9:57-75.

99. Vandenbroucke K, de Haard H, Beirnaert E, et al. Orally administered L. lactis secreting an anti-TNF Nanobody demonstrate efficacy in chronic colitis. Mucosal Immunol 2010;3:49-56.

100. Joyce SA, Shanahan F, Hill C, Gahan CG. Bacterial bile salt hydrolase in host metabolism: potential for influencing gastrointestinal microbehost crosstalk. Gut Microbes 2014;5:669-674.

101. Wall R, Cryan JF, Ross RP, Fitzgerald GF, Dinan TG, Stanton C. Bacterial neuroactive compounds produced by psychobiotics. Adv Exp Med Biol 2014;817:221-239.

102. Endersen L, Coffey A, Ross RP, McAuliffe O, Hill C, O’Mahony $\mathrm{J}$. Characterisation of the antibacterial properties of a bacterial derived peptidoglycan hydrolase (LysCs4), active against C. sakazakii and other Gram-negative food-related pathogens. Int J Food Microbiol 2015;215:79-85.

103. London LE, Kumar AH, Wall R, et al. Exopolysaccharide-producing probiotic Lactobacilli reduce serum cholesterol and modify enteric microbiota in ApoE-deficient mice. J Nutr 2014;144:1956-1962.

104. Vinogradov E, Sadovskaya I, Cornelissen A, van Sinderen D. Structural investigation of cell wall polysaccharides of Lactobacillus delbrueckii subsp. bulgaricus 17. Carbohydr Res 2015;413:93-99. 
105. Rachmilewitz D, Katakura K, Karmeli F, et al. Toll-like receptor 9 signaling mediates the anti-inflammatory effects of probiotics in murine experimental colitis. Gastroenterology 2004;126:520-528.

106. Sun Z, Harris HM, McCann A, et al. Expanding the biotechnology potential of lactobacilli through comparative genomics of 213 strains and associated genera. Nat Commun 2015;6:8322.
107. De Filippis F, Pellegrini N, Vannini L, et al. High-level adherence to a Mediterranean diet beneficially impacts the gut microbiota and associated metabolome. Gut 2016;65:1812-1821.

108. Panigrahi P, Parida S, Nanda NC, et al. A randomized synbiotic trial to prevent sepsis among infants in rural India. Nature 2017;548:407-412. 